\title{
Interworking wireless mesh networks: Problems, performance characterization, and perspectives
}

\author{
Tsai-Wei Wu ${ }^{\mathrm{a}}$, Hung-Yun Hsieh ${ }^{\mathrm{a}, \mathrm{b}, *}$ \\ ${ }^{a}$ Graduate Institute of Communication Engineering, National Taiwan University, Taipei 106, Taiwan \\ ${ }^{\mathrm{b}}$ Department of Electrical Engineering, National Taiwan University, Taipei 106, Taiwan
}

Received 8 November 2006; accepted 7 May 2007

Available online 24 May 2007

\begin{abstract}
Wireless broadband networks based on the IEEE 802.11 technology are being increasingly deployed as mesh networks to provide users with extended coverage for wireless Internet access. These wireless mesh networks, however, may be deployed by different authorities without any coordination a priori, and hence it is possible that they overlap partially or even entirely in service area, resulting in contention of radio resources among them. In this paper, we investigate the artifacts that result from the uncoordinated deployment of wireless mesh networks. We use a network optimization approach to model the problem as resource sharing among nodes belonging to one or different networks. Based on the proposed LP formulation, we then conduct simulations to characterize the performance of overlaying wireless mesh networks, with the goal to provide perspectives for addressing the problems. We find that in a system with multiple overlaying wireless mesh networks, if no form of inter-domain coordination is present, individual mesh networks could suffer from capacity degradation due to increased network contention. One solution toward addressing the performance degradation is to "interwork" these wireless mesh networks by allowing inter-domain traffic relay through provisioning of "bridge" nodes. However, if such bridge nodes are chosen arbitrarily, the problems of throughput sub-optimality and unfairness may arise. We profile the impact of bridge node selection and show the importance in controlling network unfairness for wireless mesh network interworking. We conclude that mesh network interworking is a promising direction to address the artifacts due to uncoordinated deployment of wireless mesh networks if it is supplemented with appropriate mechanisms.
\end{abstract}

(C) 2007 Elsevier Inc. All rights reserved.

Keywords: Interworking; Mesh network bridging; IEEE 802.11a/b/g; Capacity characterization; Linear programming

\section{Introduction}

Wireless mesh networking has been a subject of active investigation in recent years. Many research groups and companies have endeavored to investigate the performance benefits and drawbacks of wireless mesh networks [15,16,18]. An increasing number of citywide mesh networks as well as wireless community networks have also been deployed and put into operation all over the world [17,20,23].

A key objective of wireless mesh network deployment is to extend the coverage of wireless access while minimizing the

\footnotetext{
This work was supported in part by funds from the National Science Council under Grant NSC-94-2213-E-002-062, and the Excellent Research Projects of National Taiwan University under Grant 95R0062-AE00-04.

* Corresponding author. Department of Electrical Engineering, National Taiwan University, Taipei 106, Taiwan. Fax: +886223683824.

E-mail address: hyhsieh@cc.ee.ntu.edu.tw (H.-Y. Hsieh).
}

infrastructure cost. However, since wireless mesh networks of different scales may be deployed by different authorities without any coordination a priori, it is possible that they may overlap partially or even entirely in service area. The result is that multiple access points (APs) belonging to different network domains might be present in one area to provide service to users. For example, ${ }^{1}$ a measurement study collected from typical driving patterns in and around the Boston metropolitan area reveals that as many as 21 APs can be discovered during a successful scan [7]. The authors in [22] also show that an average number of $6.4 \mathrm{WiFi}$ networks are detected in the drive ways of a residential community. In the Electrical Engineering building

\footnotetext{
${ }^{1}$ We note that while some of the APs cited belong to last-hop wireless networks instead of wireless mesh networks, the interference problem would become even more serious if all of these APs are of the mesh type due to the increased inter-AP interferences in wireless mesh networks as we will discuss later.
} 
at the National Taiwan University campus, a typical scan will result in more than four types of networks being discovered due to the overlays of citywide mesh networks [23], campus wireless networks, and several other laboratory WiFi networks.

While the availability of multiple wireless mesh networks in one service area can potentially provide users with many access choices, it can in fact lead to serious mutual interference problem and degrade the service that can be offered to users. In [10], the authors examine the capacity of multi-hop wireless networks, and find that wireless interference among neighboring nodes is the limiting factor of the performance gain in multi-hop wireless networks. The authors in [14] study the capacity of multi-channel wireless networks, and find that with a limited number of orthogonal channels available, using multiple radios may not be capacity-efficient due to the problem with wireless interference.

Therefore, the problem of resource contention due to unplanned deployment of wireless mesh networks could lead to serious performance issue as more and more wireless mesh networks are being deployed independently. To the best of our knowledge, however, little work has been done in identifying and addressing the problem. Recently, the IEEE 802.11s task group looks into the problem of ESS (Extended Service Set) mesh networking with the goal to extend the IEEE 802.11 MAC for solving the interoperability problem across different wireless mesh networks. While several proposals have been submitted to interwork multiple 802.11s mesh networks through provisioning of Mesh Portals, the goal is to connect multiple mesh networks through efficient protocol conversion and path selection so the WLAN mesh network behaves as a traditional 802-style LAN [1,2]. The fundamental problem of performance degradation due to unplanned mesh deployment is still not yet addressed.

In this paper, we investigate the problem of resource contention among overlaying wireless mesh networks. Instead of jumping into proposing network architecture and protocol framework for mesh network interworking, we start by characterizing the fundamental performance issues of the problem with the goal to provide solution perspectives for addressing the problem in future work. Similar to related work that investigates the network capacity of wireless mesh networks $[4,12,13]$, we formulate the problem as a linear programming (LP) problem for capacity optimization of overlaying wireless mesh networks. We first present a general LP formulation for modeling a system consisting of several multi-radio, multi-channel wireless mesh networks, with each node being equipped with $802.11 \mathrm{a} / \mathrm{b} / \mathrm{g}$ multi-mode radios. The proposed formulation differs from related work $[4,12,13]$ since it explicitly formulates multi-mode heterogeneous radios and mesh network interworking. We then present simulation results to show how the proposed LP formulation can be used to shed insights into the performance issue of overlaying wireless mesh networks. We find that to mitigate the impact of wireless interference among overlaying mesh networks, one solution is to "interwork" these wireless mesh networks by allowing interdomain traffic relay through provisioning of "bridge" nodes. If such bridge nodes are chosen arbitrarily, however, the prob- lems of throughput sub-optimality and unfairness may arise. We profile the impact of bridge node selection and show the importance in controlling network unfairness for wireless mesh network interworking. Simulation results motivate research along these directions for better interworking of wireless mesh networks. The results presented in this paper can be used in tandem with related work to allow for better network architecture and protocol framework to be developed and evaluated.

The rest of this paper is organized as follows. Section 2 describes related work. Section 3 presents the system model, and Section 4 presents the LP formulation of the target problem. Section 5 presents the simulation results, and finally Section 6 concludes the paper.

\section{Related work}

In this section, we first describe the recent interest in unplanned deployment of 802.11-based wireless mesh networks. We then present several proposals from IEEE 802.11s on bridging of wireless mesh networks. Finally, we discuss related work that focuses on capacity characterization of multi-radio, multichannel wireless mesh networks using LP formulations.

\subsection{Unplanned deployment of wireless mesh networks}

Wireless mesh networks have conventionally been deployed by telecommunication service operators or site authorities under very careful deployment planning. Extensive site survey work before deployment is typically required to minimize interference and optimize coverage while incurring minimum infrastructure cost. Recently, due to the popularity of 802.11 hardware and its ease of deployment for network access, many wireless mesh networks are formed without following the traditional deployment process. Wireless community networks such as Seattle Wireless [20] and MIT Roofnet [17] have been built and put into operation in an unplanned and uncoordinated fashion, where users add or remove resources (i.e. 802.11 access point or Internet connection) from the network spontaneously.

The phenomenon of unplanned or "chaotic" deployment of wireless mesh networks has attracted the attention of several recent studies $[3,6]$. Since the placement of mesh routers in an unplanned mesh network is unconstrained, the resulting network topology is not optimized for coverage and performance. In areas with high node density, for example, serious contention among neighboring nodes may occur. Despite lack of planning and operation management, it has been shown in [6] that unplanned mesh networks still have the potential to provide users with "usable" performance. The authors in [3] propose a transmission power control algorithm to manage the transmission power levels and transmissions rates of APs and clients automatically. By reducing the transmission powers of individual mesh routers in densely packed areas, the interference can potentially be reduced and the performance can be improved.

It has to be noted that while related work has identified the existence of unplanned wireless mesh networks and the problem that results from uncoordinated deployment, the scope is limited 
to nodes belonging to one wireless mesh network (domain). It is not the focus of these studies to investigate inter-domain interaction among multiple uncoordinated wireless mesh networks that may overlap in service area. In addition, these studies have focused on performance measurements and protocol design for unplanned wireless mesh networks. This paper, on the other hand, focuses on network modeling, performance bounds, and solution perspectives for such networks.

\subsection{Bridging of wireless mesh networks}

The IEEE 802.11 ESS Mesh Networking Task Group (Task Group s) aims to define a framework for 802.11 ESS mesh networking, in particular the architecture and protocol for self-configuring paths between 802.11 APs over multi-hop topologies in an 802.11 wireless distribution system (WDS). In 802.11s, a WLAN Mesh typically consists of two or more Mesh Points interconnected via IEEE 802.11 links, and zero or more Mesh Portals. The Mesh Point is an entity with the 802.11 NIC, and the Mesh Portal is an entity at which MSDUs exit and enter a WLAN Mesh to and from other parts of a DS (distribution system) or non-802.11 network. The Mesh Point can be considered as the playing the role of the mesh router (access point), whereas the Mesh Portal can be considered as playing the role of the gateway in the literature.

Several proposals have been submitted and currently under discussion in 802.11s. The joint SEE-Mesh/Wi-Mesh proposal [1], for example, specifies protocols for unmanaged WLAN mesh networks, including path selection and optimization, interworking interoperability, power efficient operations, multimedia transport, and seamless security. In particular, the proposal considers the problem of interconnecting the WLAN Mesh with other 802 networks through Mesh Portals. Since the WLAN Mesh in $802.11 \mathrm{~s}$ is a layer-2 network, it functions as a traditional IEEE 802-style LAN and needs to support transparent multi-hop broadcast and unicast delivery from higher layer protocols. To achieve this objective, the Mesh should be able to interconnect with other networks using both layer-2 bridging (where the Mesh Portal acts as a bridge) and layer-3 internetworking (where the Mesh Portal acts as a gateway router).

Another proposal called SnowMesh [2] considers the problems of backward compatibility with existing 802.11 STAs, and proposes protocols in the area of routing, security, QoS, and power saving for the WLAN Mesh. In particular, the proposal discusses the problems of partitioned Mesh and Mesh merging. Two partitioned Meshes may be merged by the Mesh Portal that can communicate with both Meshes if their operational modes and parameter settings (e.g. the physical layer technology and routing protocols) are compatible. The Mesh Portal can then associate with one member of the other Mesh and relay traffic between the two Meshes.

Although IEEE 802.11s has considered the problem of interworking WLAN mesh networks, it focuses on the aspects of framework design and protocol development. The work presented in this paper, on the other hand, focuses on related optimization issues, such as the placement of Mesh
Portals. Hence, the results presented in this paper can provide guidelines to be used in tandem with 802.11s proposals (or specifications) for practical framework and protocol design considerations.

\subsection{Capacity characterization of wireless mesh networks}

Several studies exist in the literature that use the approach of network optimization to characterize the capacity of wireless mesh networks. For example, the authors in [12] formulate a multi-commodity flow problem augmented with wireless interference constraints to compute the optimal throughput that can be supported between any given pairs of source and destination nodes in a single-radio wireless mesh network. They show that the problem of finding the optimal throughput is NPhard, and then present approaches for computing the upper and lower bounds of the optimal throughput. In [13], the authors propose a network model for characterizing the capacity of multi-radio, multi-channel wireless mesh networks. They design a fast primal-dual algorithm for a fully polynomial time approximation solution (FPTAS) and use it to derive an upper bound of the achievable throughput. Two link channel assignment schemes are developed for deriving the lower bounds of the problem. In [4], the authors consider a similar problem for multi-radio wireless mesh networks using LP. They formulate the joint channel assignment and routing problem while taking into account the interference constraints, the number of channels in the network, and the number of radios available at each mesh router. The formulation is used to develop a solution that optimizes the overall network throughput subject to fairness constraints on allocation of scarce wireless capacity. In [8], the authors propose two mixed integer LP models for solving the fixed channel assignment problem and they implement a variant of the greedy sequential heuristic algorithm for finding maximal cliques in the link interference model.

While these studies pave the foundation of our work, they consider only homogeneous radio systems in one mesh network, where all nodes in the network use the same mode of operations. This paper, on the other hand, considers $802.11 \mathrm{a} / \mathrm{b} / \mathrm{g}$ multi-mode radio systems for modeling the mesh nodes, and, more importantly, investigates in a unified framework the optimization for interworking of multi-mode, multi-radio, multichannel wireless mesh networks.

\section{System model}

We consider wireless mesh networks based on the IEEE $802.11 \mathrm{a} / \mathrm{b} / \mathrm{g}$ technology, where individual mesh routers may be equipped with one or more multi-mode radios based on 802.11a, 802.11b, 802.11g, or any combination thereof. Each mode supports multi-channel, multi-rate (rate-adaptive) operations as specified in the IEEE 802.11 standards.

As shown in Fig. 1, wireless mesh networks deployed by different authorities may overlap partially or even entirely in service area. Existing formulations, however, focus only on the scenario where there is only one wireless mesh network in the system. To model a system with potentially multiple 


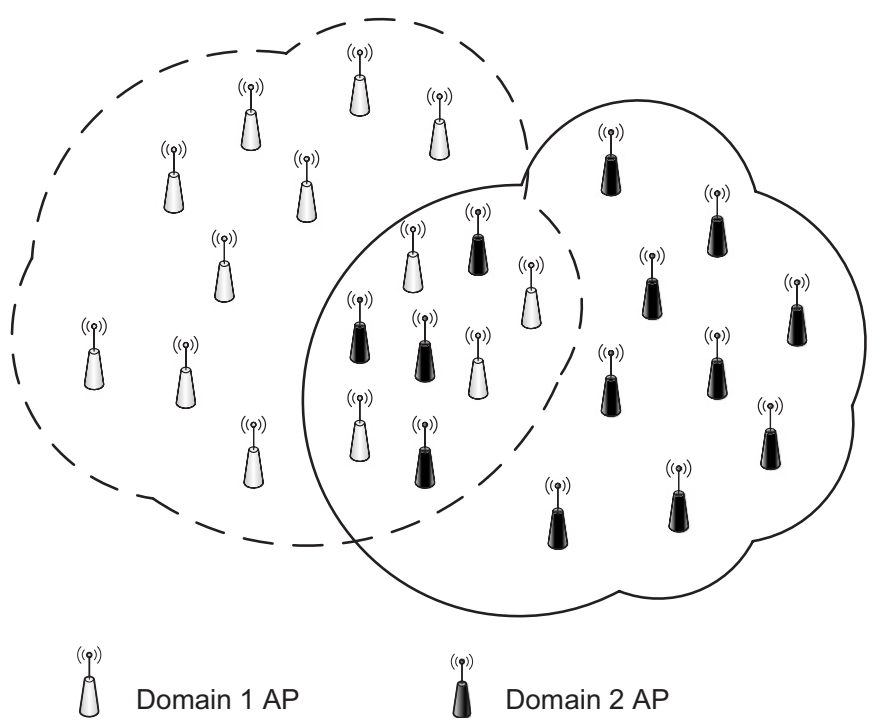

Fig. 1. Two wireless mesh networks with service area overlap.

wireless mesh networks, we introduce the concept of "network domain" in our formulation. Let $G=(V, E)$ be a connected graph representing the whole system, with $V$ being the set of mesh routers and $E$ being the set of directed links. Each node $u$ is then tagged with a domain variable $\delta_{u}$ depending on which network domain it belongs to. Given appropriate constraints on inter-domain communications, we can then use one wireless mesh network with multiple domains to model a system with multiple wireless mesh networks. In the following, we describe in details how the nodes and links are modeled.

\subsection{Node model}

A multi-mode $802.11 / \mathrm{a} / \mathrm{b} / \mathrm{g}$ mesh node can be tuned to any mode for communication with nearby nodes. Depending on hardware configuration, however, these modes can be activated "simultaneously" or only "one at a time." For example, Atheros AR5002X and AR5002AP-2X are both multi-mode $802.11 \mathrm{a} / \mathrm{b} / \mathrm{g}$ WLAN chipsets, but the former can be tuned to one mode at a time, whereas the latter can support concurrent 802.11a and 802.11b/g operations [5]. Therefore, to model a multi-mode node, it is necessary to consider the type of WLAN chipsets involved. In this paper, we use $F_{u}$ to denote the set of modes ( 0 for $802.11 \mathrm{~b}, 1$ for $802.11 \mathrm{~g}$, and 2 for 802.11a) that node $u$ supports, and $\pi_{u}$ to denote the "effective" number of radios that node $u$ has. For example, assume that node $u$ is based on the AR5002X chipset, and node $v$ is based on the AR5002AP-2X chipset. Then $\pi_{u}=1, \pi_{v}=2$, and $F_{u}=F_{v}=$ $\{0,1,2\}$. Note that in this formulation, a two-radio node $u$ with two AR5002AP-2X radios will be modeled as $\pi_{u}=4$.

Another issue that needs to be considered in modeling a multi-mode node is the backward compatibility of $802.11 \mathrm{~g}$ radio with $802.11 \mathrm{~b}$ [11]. Hence, in our model, even if node $u$ is equipped only with the $802.11 \mathrm{~g}$ radio, we still set $F_{u}=\{0,1\}$, the same as one with $802.11 \mathrm{~b} / \mathrm{g}$. We show in
Table 1

A category of multi-mode radio

\begin{tabular}{lll}
\hline Types of radios & $\begin{array}{l}\text { Number of radios } \\
\pi_{u}\end{array}$ & $\begin{array}{l}\text { Communication modes } \\
F_{u}\end{array}$ \\
\hline Single radio, b only & 1 & $\{0\}$ \\
Single radio, g only & 1 & $\{0,1\}$ \\
Single radio, a only & 1 & $\{2\}$ \\
Single radio, a/b & 1 & $\{0,2\}$ \\
Single radio, a/b/g & 1 & $\{0,1,2\}$ \\
Two radios, a and b & 2 & $\{0,2\}$ \\
Two radios, a and b/g & 2 & $\{0,1,2\}$ \\
\hline
\end{tabular}

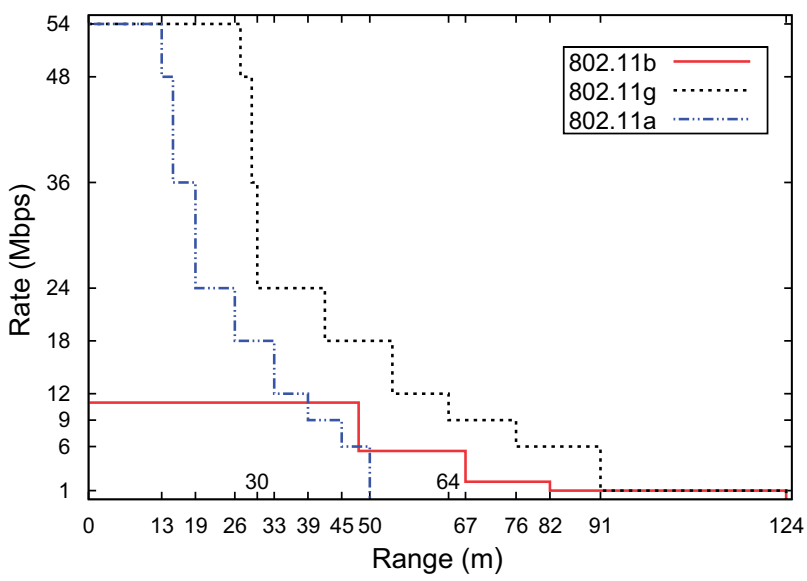

Fig. 2. IEEE $802.11 \mathrm{a} / \mathrm{b} / \mathrm{g}$ rate-range distribution.

Table 1 different types of multi-mode radios popularly used today.

\subsection{Link model}

We use $M=\{0,1,2,3, \ldots, 17\}$ to denote the set of channels available, where $M B=\{0,1,2\}$ denotes the three orthogonal channels operated by IEEE $802.11 \mathrm{~b}, M G=\{3,4,5\}$ denotes the three orthogonal channels operated by IEEE $802.11 \mathrm{~g}$, and $M A=\{6,7,8, \ldots, 17\}$ denotes the 12 orthogonal channels operated by IEEE $802.11 \mathrm{a}$. Recall that $802.11 \mathrm{~g}$ radio is backward compatible with $802.11 \mathrm{~b}$. Hence an $802.11 \mathrm{~g}$ radio can use at most six channels $M G \cup M B=\{0,1,2,3,4,5\}$. Note that while $802.11 \mathrm{~b}$ and $802.11 \mathrm{~g}$ in fact operate at the same frequency bands, having different sets of channel variables for $M B$ and $M G$ allows us to model different rate-range relationships that $802.11 \mathrm{~b}$ and $802.11 \mathrm{~g}$ exhibit as discussed in the following.

In our link model, a link $l_{u v} \in E$ from node $u$ to node $v$ exists only if nodes $u$ and $v$ share at least one common mode (i.e. $F_{u} \cap F_{v}$ is non-empty), and the distance $d_{u v}$ between node $u$ and node $v$ is within the mutual transmission range. As shown in Fig. 2, there is an inverse relationship between range and rate as governed by the channel propagation model. In our model, the highest data rate that a mode (or channel) can operate over the given distance is used as the capacity of the given mode 
(or channel). For example, when the distance $d_{u v}$ between node $u$ and node $v$ is equal to $46 \mathrm{~m}$ and $F_{u} \cap F_{v}=\{0,1,2\}$, the three data rates that $l_{u v}$ could support in different modes are 6,11 , and $18 \mathrm{Mbps}$, respectively.

\subsection{Interference model}

We consider an interference model that reflects the DATAACK handshake used popularly in existing 802.11 based wireless mesh networks [4,13], where a successful transmission requires both the sender and the receiver to be free of interference. In our model, we assume that each node uses the same power for packet transmissions, and the interference range $R^{I}$ is fixed. Two different links can transmit on a common channel simultaneously if both the sender and the receiver of the other link is at least $R^{I}$ apart from each other. For example, assume that nodes $u, v, x$, and $y$ are separated such that $d_{u v}$ and $d_{x y}$ are within the mutual transmission range. Then links $l_{u v}$ and $l_{x y}$ can be active at the same time if and only if $d_{u x} \geqslant R^{I}$, $d_{u y} \geqslant R^{I}, d_{v x} \geqslant R^{I}$, and $d_{v y} \geqslant R^{I}$.

Note that since $802.11 \mathrm{~g}$ and $802.11 \mathrm{~b}$ operate at the same frequency band, $M B$ and $M G$ are not necessarily orthogonal even though different physical layer technologies may be used. The authors show in $[9,19]$ that there is indeed interference between IEEE $802.11 \mathrm{~b}$ and $802.11 \mathrm{~g}$ radios. Therefore, in our interference model, we assume that channels in $M B$ can interfere with channels in $M G$. Let $I_{k}$ be the set of interfering channels for channel $k$. Then $I_{0}=\{0,3\}, I_{4}=\{1,4\}$, and $I_{7}=\{7\}$. Specifically, assume that $l_{u v}$ and $l_{x y}$ are two distinct links with $l_{u v}$ operating on channel 0 and $l_{x y}$ operating on channel 3. If any of $d_{u x}, d_{u y}, d_{v x}$ and $d_{v y}$ is less than $R^{I}$, then these two links interfere with each other. The same is true for the case when $l_{u v}$ operates on channel 1 and $l_{x y}$ operates on channel 4 , and the case when $l_{u v}$ operates on channel 2 and $l_{x y}$ operates on channel 5. We use $E_{u v}^{I}$ to denote the set of links interfering with link $l_{u v}\left(E_{u v}^{I}\right.$ includes $l_{u v}$ itself).

\subsection{Interworking model}

We assume that in the absence of coordination, mesh nodes belonging to different domains do not communicate with each other. That is, link $l_{u v}$ does not exist if nodes $u$ and $v$ belong to different domains. To allow for interworking, we introduce the concept of "bridge" nodes that can facilitate inter-domain communication. A bridge node may need to be equipped with multiple interfaces (to relay between, say, 802.11a and 802.11b networks) or it may need to perform protocol conversion between neighboring network domains [1,2]. For example, as shown in Fig. 3, without the presence of the bridge node br, traffic from node $\mathbf{s} \mathbf{1}$ has to traverse along route $\mathbf{R} \mathbf{1}$ to reach the gateway node gw1. If, on the other hand, node br is made to relay traffic for nodes in domain 1 , then a shorter and less congested route R1' can be taken between nodes $\mathbf{s} 1$ and gw1. We use $\beta_{u}$ to denote whether node $u$ is a bridge node or not. Node $u$ can communicate with nodes in different domains only when $\beta_{u}$ is 1 .

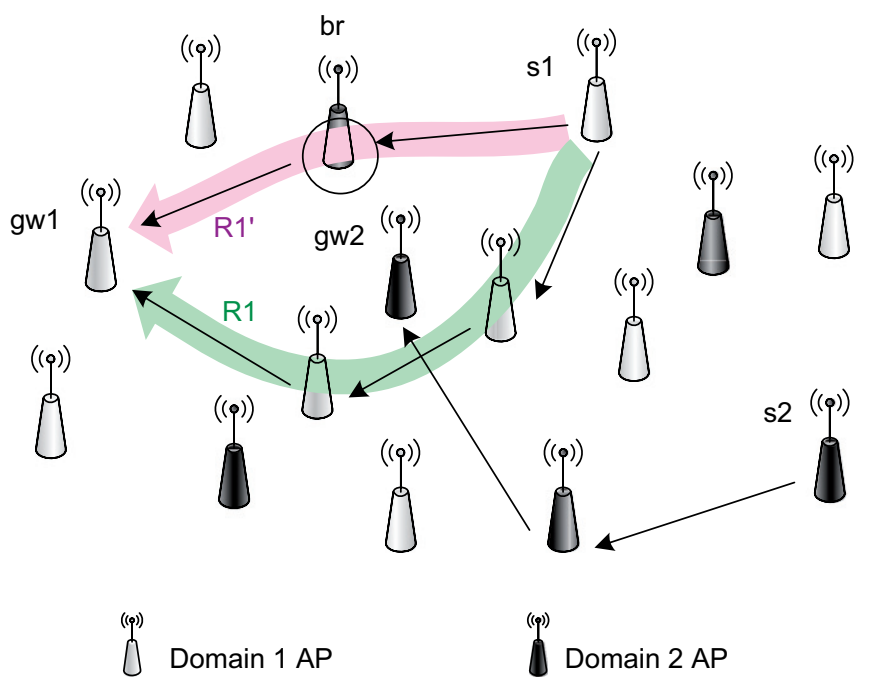

Fig. 3. Illustration of bridge nodes.

\section{LP formulation}

We use an LP formulation to find the capacity region of overlaying wireless mesh networks. First, we create $\left|M_{u v}\right|$ virtual links on link $l_{u v}$, where $M_{u v}$ denotes the set of channels that can be used on link $l_{u v}$ (i.e. channels that both nodes $u$ and $v$ can use). The capacity of the virtual link $l_{u v}$ on channel $k$ is $c_{u v}^{k}$ as determined by the link model described in Section 3.2. We consider the scenario where there are multiple sourcedestination pairs in the network, and each pair has a unique connection ID. Flow rate $f_{u v}^{k q}$ denotes the amount of flow over virtual link $l_{u v}$ on channel $k$ for connection $q$. A virtual super sink $t$ to which every destination node has a directed link is created. The bandwidth between each destination node and the virtual sink $t$ is assumed to be infinite, meaning that the bottleneck links are in the wireless domain. Table 2 lists the symbols used in the formulation.

To find the maximal network capacity in our system model using LP optimization, we proceed through the following formulations:

Objective function: A simple objective function to maximize the amount of outgoing flows for individual connections may lead to severe bias on bandwidth allocation among source nodes. Therefore, in our formulation we assume that each source node $u$ has a traffic demand $\Phi(u)$, and we introduce a scaling factor $\gamma$ for capacity maximization [13]. In this way, the outgoing traffic for each connection is at least $\gamma \Phi(u)$, and the goal is to maximize the scaling factor $\gamma$ in our formulation. Note that a connection may get higher throughput than $\gamma \Phi(u)$ as long as such rate allocation does not prevent other connections from meeting their traffic demands. Other objective functions that consider more sophisticated fairness models (e.g. max-min fairness model) as proposed by Tang et al. [21] can also be used in our formulation.

Flow conservation: At every node, except the source and the destination, the amount of incoming flow should be equal to the amount of outgoing flow. We assume that the gateway node 
Table 2

Index of symbols used in the formulation

\begin{tabular}{ll}
\hline Symbols & Definition \\
\hline$G$ & connected directed graph \\
$V$ & set of vertices in $G$ \\
$E$ & set of directed links in $G$ \\
$E^{I}$ & set of interfering node pairs in $G$ \\
$E_{u}$ & set of links incident on node $u$ \\
$\pi_{u}$ & number of radios on node $u$ \\
$\pi_{u}^{n}$ & number of radios supporting communication mode $n$ on node $u$ \\
$M_{n}$ & set of channels that can be used by radio of mode $n$ \\
$M_{u v}$ & set of channels that can be used on link $l_{u v}$ \\
$d_{u v}$ & distance between node $u$ and node $v$ \\
$l_{u v}$ & directed link from node $u$ to node $v$ \\
$c_{u v}^{k}$ & capacity of link $l_{u v}$ on channel $k$ \\
$f_{u v}^{k q}$ & flow over link $l_{u v}$ on channel $k$ by connection $q$ \\
$I_{k}$ & set of channels interfering with channel $k$ (includes $k$ ) \\
$E_{u v}^{I}$ & set of links interfering with link $l_{u v}$ (includes $\left.l_{u v}\right)$ \\
$S$ & set of source nodes \\
$D$ & set of destination nodes \\
$Q$ & set of connections, $Q=\{1,2, \ldots,|S|\}$ \\
$\hat{q}$ & the source node of connection $q$ \\
$\vec{s}$ & the connection from source node $s$ \\
$\delta_{u}$ & domain of node $u$ \\
$\beta_{u}$ & whether node $u$ is a bridge node \\
\hline &
\end{tabular}

only forwards traffic to virtual super node $t$ without relaying any traffic to other nodes. Let $E_{u, o}$ denote the set of outgoing links from node $u$ and $E_{u, i}$ denote the set of incoming links to node $u$. Hence $E_{u}=E_{u, o} \cup E_{u, i}$ denotes the set of links incident on node $u$. The constraints for flow conservation can be formulated as follows:

$$
\begin{gathered}
\sum_{l_{x u} \in E_{u, i}} \sum_{k \in M_{x u}} f_{x u}^{k q}=\sum_{l_{u y} \in E_{u, o}} \sum_{k \in M_{u y}} f_{u y}^{k q} \\
\forall q \in Q, u \in V \backslash\{D, \hat{q}, t\}, \\
\sum_{l_{s y} \in E_{s, o}} \sum_{k \in M_{s y}} f_{s y}^{k \vec{s}}=\gamma \Phi(s) \quad \forall s \in S, \\
\sum_{l_{x s} \in E_{s, i}} \sum_{k \in M_{x s}} f_{x s}^{k \vec{s}}=0 \quad \forall s \in S, \\
\sum_{l_{x d} \in E_{d, i}} \sum_{k \in M_{x d}} \sum_{q \in S} f_{x d}^{k q}=f_{d t} \quad \forall d \in D, \\
\sum_{d \in D} f_{t d}=0 .
\end{gathered}
$$

Radio constraint: Since node $u$ has $\pi_{u}$ radios, it can use at most $\pi_{u}$ radios at a time for transmission and/or reception. Define $\pi_{u}^{n}$ as the number of radios on node $u$ that support communication mode $n \in F_{u}$. (For example, node $u$ with a single $\mathrm{a} / \mathrm{b} / \mathrm{g}$ radio has $\pi_{u}^{0}=\pi_{u}^{1}=\pi_{u}^{2}=1$.) Let $M_{n}$ be the set of channels that mode $n$ radio operates, then $M_{0}=M B$,

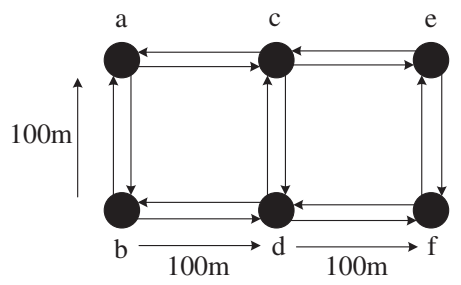

Fig. 4. A simple communication graph.

$M_{1}=M B \cup M G$, and $M_{2}=M A$ as defined in Section 3.2. We have the following radio constraints:

$$
\begin{gathered}
\sum_{l_{x y} \in E_{u}} \sum_{k \in M_{x y}} \frac{\sum_{q \in Q} f_{x y}^{k q}}{c_{x y}^{k}} \leqslant \pi_{u} \quad \forall u \in V, \\
\sum_{l_{x y} \in E_{u}} \sum_{k \in M_{x y} \cap M_{n}} \frac{\sum_{q \in Q} f_{x y}^{k q}}{c_{x y}^{k}} \leqslant \pi_{u}^{n} \quad \forall u \in V, \quad n \in F_{u} .
\end{gathered}
$$

Interference constraint: For ease of formulation, we define $E^{I}$ as the set of interfering node pairs in $G$. A given node pair $(u, v)$ belongs to $E^{I}$ if and only if $d_{u v}>R^{T}$ and $d_{u v} \leqslant R^{I}$, where $R^{T}$ is the transmission range and $R^{I}$ is the interference range. Therefore, one way to model the wireless interference constraint is:

$$
\sum_{l_{x y} \in E_{u} \cup E_{v}} \sum_{h \in I_{k}} \frac{\sum_{q \in Q} f_{x y}^{h q}}{c_{x y}^{h}} \leqslant 1 \quad \forall l_{u v} \in E \cup E^{I}, \quad k \in M_{u v} .
$$

However, the constraint in Eq. (8) does not include all potential interferences, and hence the resultant network capacity is just an upper bound as shown in [13]. A lower bound of the network capacity thus needs to be found to cap the actual network capacity of the network. Recall that $E_{u v}^{I}$ denotes the set of links interfering with link $l_{u v}\left(E_{u v}^{I}\right.$ includes $\left.l_{u v}\right)$. If we allow only one link in the set $E_{u v}^{I}$ to be active at one time, then such a constraint places a lower bound on the achievable network capacity [12]. For example, it is possible that links $l_{1}$ and $l_{2}$ interfere with link $l_{0}$, but they do not interfere with each other. If only $l_{1}$ or $l_{2}$ is allowed to transmit at given time, then the achievable capacity is a conservative estimate. Therefore, the lower bound of the network capacity can be obtained using the following interference constraint:

$$
\sum_{l_{x y} \in E_{u v}^{I}} \sum_{h \in I_{k}} \frac{\sum_{q \in Q} f_{x y}^{h q}}{c_{x y}^{h}} \leqslant 1 \quad \forall l_{u v} \in E, \quad k \in M_{u v} .
$$

We now give a simple example in Fig. 4 to explain the set of interference links involved in Eqs. (8) and (9) and the bounds derived therein. Assume all nodes in Fig. 4 have the transmission range $R^{T}=120 \mathrm{~m}$, and the interference range $R^{I}=180 \mathrm{~m}$. The interference set of link $l_{d c}$ for deriving the upper bound is shown in Fig. 5(a). Since only links $l_{a b}, l_{b a}, l_{e f}$, and $l_{f e}$ do not share any endpoint with link $l_{d c}$, in Eq. (8) the interference set of link $l_{d c}$ will not include links $l_{a b}, l_{b a}, l_{e f}$, and $l_{f e}$. On the other hand, the interference set of link $l_{d c}$ for deriving the 


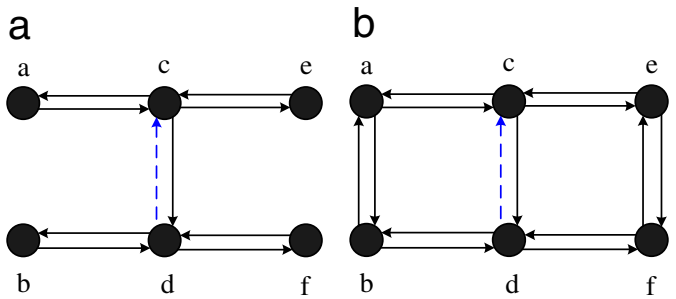

Fig. 5. Illustration of interference links. (a) Interference set for link $l_{d c}$ (upper bound); (b) interference set for link $l_{d c}$ (lower bound).

lower bound is shown in Fig. 5(b). Since all links interfere with link $l_{d c}$, in Eq. (9), the interference set of link $l_{d c}$ will involve all other links in the networks. It is obvious from Fig. 5 that for the upper bound, the constraint does not include all links that will interfere with link $l_{d c}$ such as $l_{a b}$ and $l_{e f}$. For the lower bound, the constraint excludes the possibility for links $l_{a b}$ and $l_{e f}$ to transmit at the same time. Therefore, Eqs. (8) and (9) only place the upper bound and lower bound of the actual interference constraint, respectively. As discussed in [12], it is possible to use the concept of maximal independent sets and cliques in the conflict graph of Fig. 4 for better capturing the wireless interference. However, since finding all maximal independent sets and/or cliques is an NP-complete problem [12], in this paper we use the two bounds to profile the actual capacity of the system.

Interworking constraint: A key constraint in interworking multiple wireless mesh networks is to limit the number of bridge nodes $B$ while maximizing the total network capacity. In our formulation, nodes belonging to different domains can communicate with each other only if at least one of them is a bridge node. Otherwise, the link $l_{u v}$ does not exist if nodes $u$ and $v$ belong to different domains. Let $\beta_{u}$ denote whether node $u$ is a bridge node or not, then the interworking constraints can be formulated as follows:

$$
\begin{gathered}
\sum_{q \in Q} f_{u v}^{k q} \leqslant c_{u v}^{k} \beta_{u} \quad \forall l_{u v} \in E, \quad k \in M_{u v}, \quad \text { if } \delta_{u} \neq \delta_{v}, \\
\sum_{q \in Q} f_{u v}^{k q} \leqslant c_{u v}^{k} \quad \forall l_{u v} \in E, \quad k \in M_{u v}, \text { if } \delta_{u}=\delta_{v}, \\
\sum_{u \in V} \beta_{u} \leqslant B \\
\beta_{u} \in\{0,1\} \quad \forall u \in V .
\end{gathered}
$$

The final LP formulation for finding the upper bound and lower bound of the network capacity can be obtained by combining the flow conservation constraint, radio constraint, interference constraint (one for each bound), and interworking constraint altogether.

\section{Simulation results}

In this section, we present simulation results to show how the proposed LP formulation can be used for characterizing and

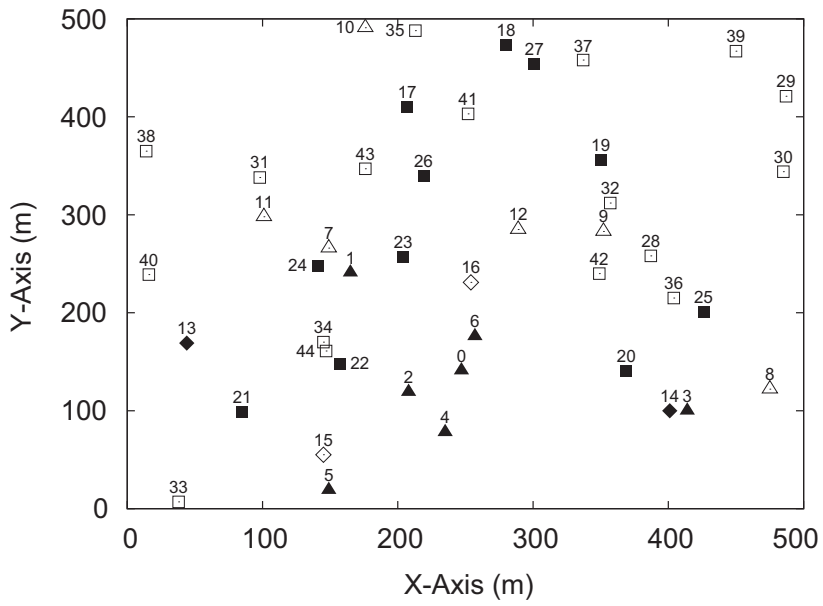

Fig. 6. Two overlaying wireless mesh networks.

providing insights to the problem of wireless mesh network interworking.

\subsection{Overlaying networks without coordination}

We consider two arbitrary $802.11 \mathrm{~b}$ mesh networks with 20 nodes (b1) and 25 nodes (b2), respectively, in a $500 \times 500$ area. As shown in Fig. 6, nodes in black belong to network b1 while nodes in white belong to network b2. Seven and six nodes are randomly selected from networks b1 and b2, respectively, as the source nodes (indicated by triangles). Two wired nodes from each of the two networks are designated as gateway nodes (indicated by diamonds) that can be used as the traffic sink for the source nodes in the same network. The rest of the nodes are mesh routers (indicated by squares) for traffic relay between source and gateway nodes. More specifically, in Fig. 6, nodes 0-6 are source nodes of network b1, and nodes 7-12 are source nodes of network b2. Nodes 13 and 14 are gateway nodes of network b1, and nodes 15 and 16 are gateway nodes of network b2. We assume that there is a minimum traffic demand $\gamma_{1}$ and $\gamma_{2}$ (to be maximized through network optimization) for source nodes in networks b1 and b2, respectively. That is, $\Phi(u)$ in Section 4 is set to 1 for each source node $u$.

To understand the impact of network overlays, we first consider the scenario where only network b1 is present in the $500 \times 500$ area (all nodes belonging to network b2 are removed), and find through LP optimization the maximum traffic demand (throughput) $\gamma_{1}$ that can be achieved. We then consider an opposite scenario where only network b2 is present. Finally, we include all 45 nodes in the area, and find the maximum traffic demand $\gamma$ that can be achieved for the two overlaying networks. We require the throughputs of all source nodes (be it of network b1 or b2) to be greater than or equal to $\gamma$ for fairness. (We consider the scenario where networks b1 and b2 can have different throughputs later in this section.) No inter-domain traffic is allowed for this scenario, where source nodes in network b1 (or b2) can use only the mesh routers and gateway nodes in network b1 (or b2) for traffic relay. This scenario considers the 
a

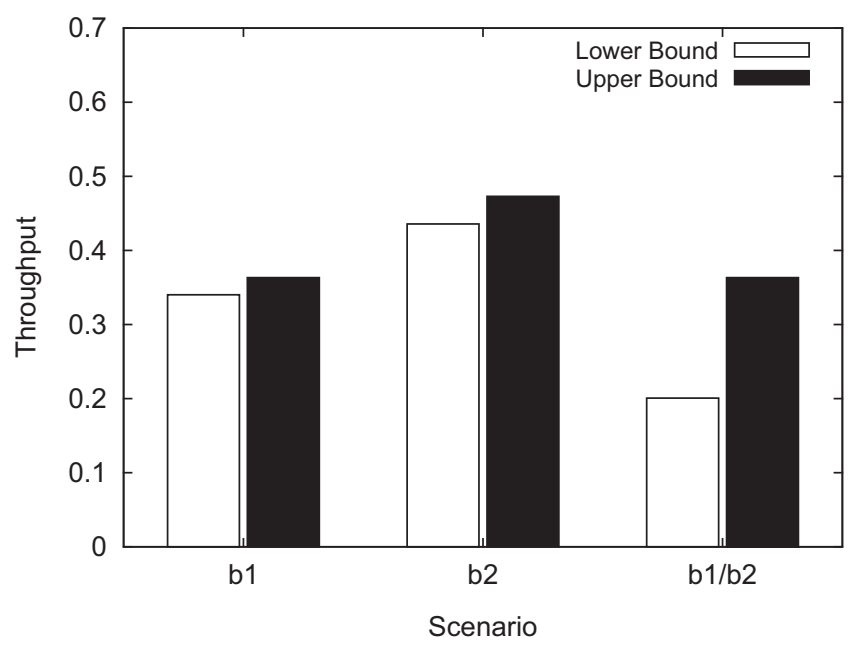

b

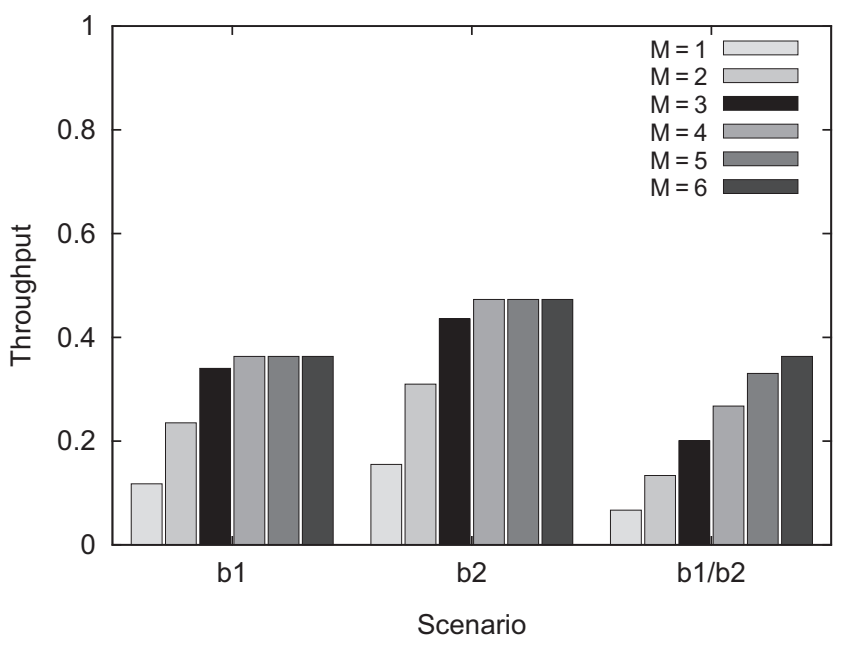

Fig. 7. Impact of network contention. (a) Throughput degradation; (b) number of channels (lower bound).

artifacts of mesh network deployment where two wireless mesh networks are deployed independently by different authorities over the same geographic region.

Fig. 7(a) thus shows the values of the maximum achievable traffic demand for the three different scenarios as labeled by $\mathrm{b} 1, \mathrm{~b} 2$, and $\mathrm{b} 1 / \mathrm{b} 2$, respectively. It is clear from the figure that scenario $\mathrm{b} 1 / \mathrm{b} 2$ has the worst per-connection performance compared to the two scenarios when only one network is present. The reason is due to the increased wireless interference among mesh nodes from different networks (i.e. network contention) and the requirement that all source nodes should meet the same minimum traffic demand.

Evidently, the performance limitation due to wireless interference can be alleviated by allocating more orthogonal channels for mesh nodes to operate. In Fig. 7(b), we show the performance of the three different scenarios when the number of channels increases from 1 to 6 (the default case has three channels). Due to limited space, only the lower bound is shown in the figure. We can observe from Fig. 7(b) that increasing the number of orthogonal channels can potentially alleviate the impact of wireless interference and increase the maximum achievable throughput. For example, consider the performance in scenarios $\mathrm{b} 1$ and $\mathrm{b} 1 / \mathrm{b} 2$ when there are six orthogonal channels. Since four channels are sufficient for network b1 to achieve the optimal performance, the overlay of network b2 in the same region does not cause the performance of network b1 to degrade due to the additional channels that can be used to avoid interference (the throughput in scenario $\mathrm{b} 1 / \mathrm{b} 2$ for $M=6$ is the same as that in scenario b1 for $M=4$ ). Therefore, it is conceivable that for the same network topology and traffic load, the impact of network overlay is less serious for two 802.11a mesh networks compared to $802.11 \mathrm{~b}$ since the former can have as many as 12 orthogonal channels in the $5 \mathrm{GHz}$ U-NII band. Despite, note that having more orthogonal channels does not do away the problem of performance degradation due to network overlays. As can be seen from Fig. 7(b),

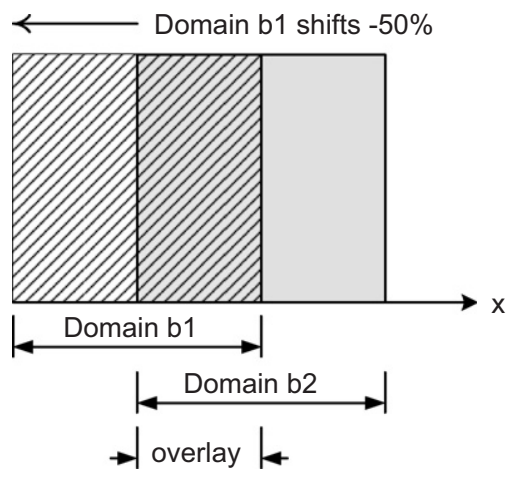

Fig. 8. Illustration of the overlay region.

it requires more channels for nodes in network b2 to restore its throughput when network b1 is overlaid in the same area (the throughput in scenario b1/b2 is less than that in scenario b2 for $M=6$ ). The size of the network, the distribution of nodes, the load of the traffic, and the level of interference will all contribute to the demand for more orthogonal channels.

Fig. 7 considers the case when two wireless mesh networks overlap entirely over the same geographic region. In practice, however, it is possible that two independently deployed mesh networks are only partially overlapped. To understand the problem of network overlay due to partial overlap, we fix the locations of nodes in network b2, and shift those of network b1 horizontally as shown in Fig. 8. Network b2 occupies a $500 \times 500$ area bounded by coordinates $(0,0)$ and $(500,500)$ in the $x-y$ plane. Network b1, on the other hand, occupies another equally sized area bounded by $(x, 0)$ and $(500+x, 500)$. We change the value of $x$ from -800 to 800 so the relationship of the two networks vary from non-interfering $(x=-800$ where no node in network b1 is within the transmission range of nodes in network b2 and vice versa) to maximum interference $(x=0)$ and to non-interfering $(x=800)$ again. Such a 
a

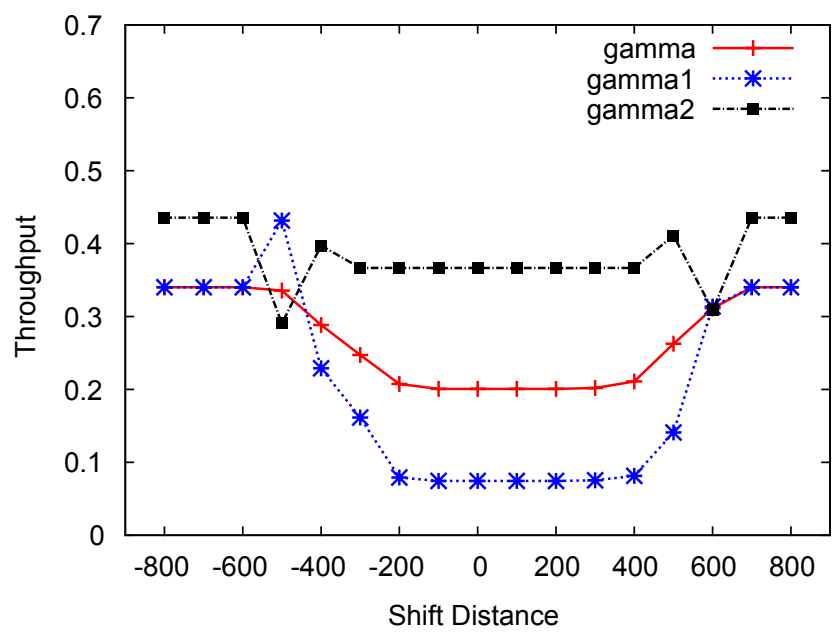

b

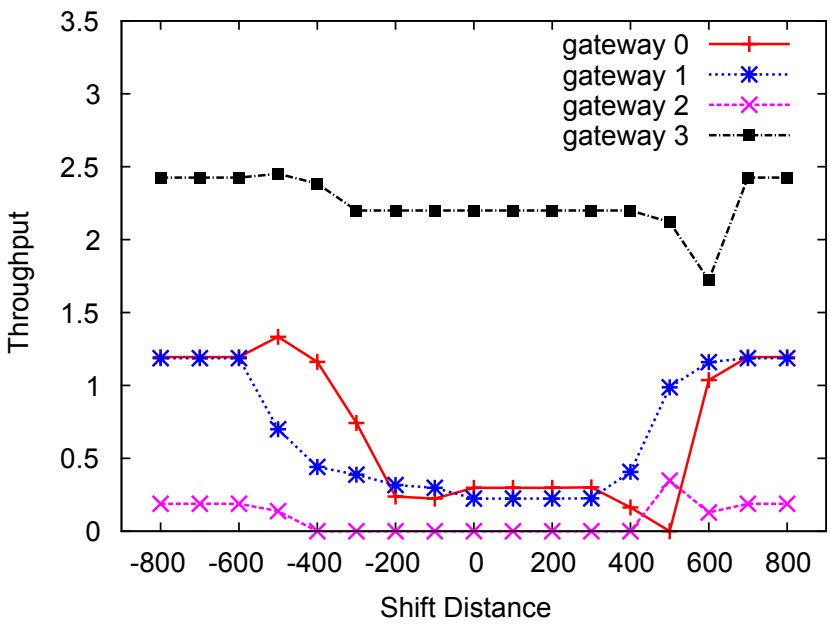

Fig. 9. Impact of partial overlap. (a) Throughput of source nodes; (b) load at different gateway nodes.

configuration makes it possible to investigate the impact of partial overlap on the performance of two overlaying networks.

In Fig. 9(a), we show the maximum throughput achievable at each source node (for clarity only the lower bound is shown). We consider two scenarios: in the first scenario the throughputs of all 13 source nodes should meet the same minimum traffic demand $\gamma$, while in the second scenario the source nodes of networks b1 and b2 can have different minimum throughputs $\gamma_{1}$ and $\gamma_{2}$, respectively, for sake of capacity optimization. We observe that if both networks are required to have the same minimum throughput $\gamma$, then as the region of overlap increases, the maximum achievable throughput decreases. Even when the two networks are only partially overlapped, the degradation in throughput is still non-trivial. If, on the other hand, networks b1 and $\mathrm{b} 2$ are allowed to have different minimum throughputs for network optimization, the performance of individual networks may suffer differently as the region of overlap increases. For example, network b1 has $78 \%$ throughput degradation when the overlap region is between $60 \%$ and $100 \%$ of the network dimension. Note that the throughput degradation of network b1 is more severe than that of network b2, and there is significant unfairness between the two networks (consider the values of $\gamma_{1}$ and $\gamma_{2}$ when the shift distance is between -200 and +400 ) when the two networks experience significant interfere with each other.

It can be observed that the curves in Fig. 9 are not symmetric to the point $x=0$. The reason is due to the asymmetric distribution of nodes in individual networks as can be shown from Fig. 9(b), where the traffic flows at each gateway node with respect to the value of $x$ are plotted. Consider, for example, the values of $\gamma_{1}$ at $x=-400$ and 400 . In the former case, gateway 13 suffers from less interference from nodes in network b2 compared to the latter case, and hence it can accommodate more traffic flows for nodes in network b1 (as can be seen in Fig. 9(b)), resulting in $\gamma_{1}$ at $x=-400$ being higher than that at $x=400$.

\subsection{Overlaying networks with coordination}

We have seen from Section 5.1 that if two wireless mesh networks are deployed independently but overlap in the service area, then the performance of individual networks will suffer due to the increased wireless interference. The problem will exist even if the two networks are only partially overlapped, and it cannot be completely addressed by simply allocating more orthogonal channels for use. In this section, we show the benefits of network coordination in addressing the problem.

To allow for inter-domain traffic between overlaying wireless mesh networks, we introduce $B$ bridge nodes in the network topology of Fig. 6. Bridge nodes can relay inter-domain traffic in addition to intra-domain traffic, and they are selected from existing mesh nodes either randomly or through network optimization depending on the scenario considered. We require all source nodes from networks b1 and b2 to achieve the same minimum throughput, and we vary the number of bridge nodes selected to find the change in the maximum achievable throughput. Fig. 10(a) shows the impact of the number of bridge nodes when $B$ bridge nodes are randomly chosen. When the number of bridge nodes vary from one node to 45 nodes, the upper bound is improved up to $143 \%$ while the lower bound is improved up to $178 \%$. Compared with the throughputs of scenarios b1, b2, and b1/b2 in Fig. 7(a), it can be observed that with the support of about 15 bridge nodes, all source nodes in the overlaying networks can achieve higher throughputs than what they could achieve in individual networks. That is, instead of suffering from throughput degradation due to network contention, the two overlaying networks can benefit from network coordination with support from the bridge nodes for relaying inter-domain traffic.

The bridge nodes in Fig. 10(a) are chosen randomly without any consideration of network optimization, and hence it can be observed that the curve has a stair-step shape. This implies that in some cases introducing more bridge nodes may have no 
a

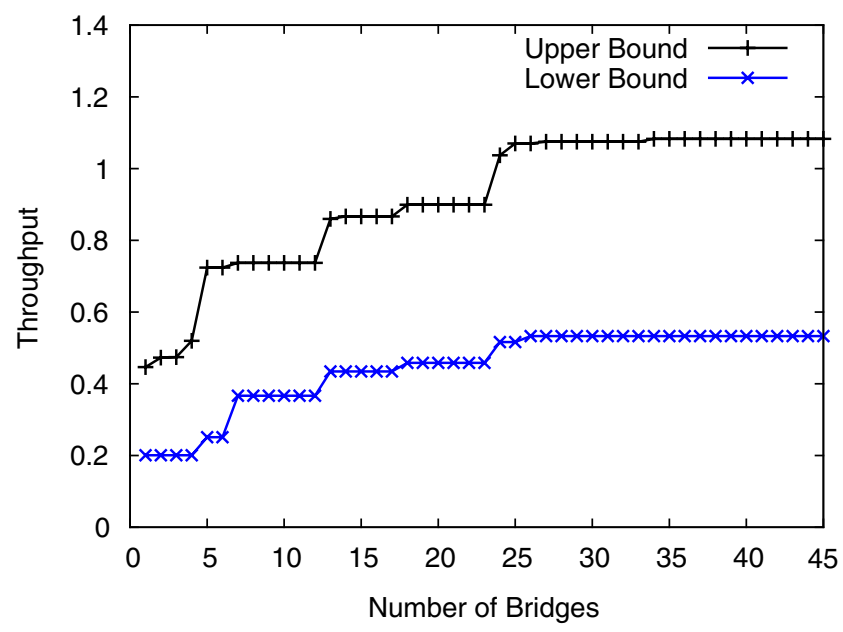

b

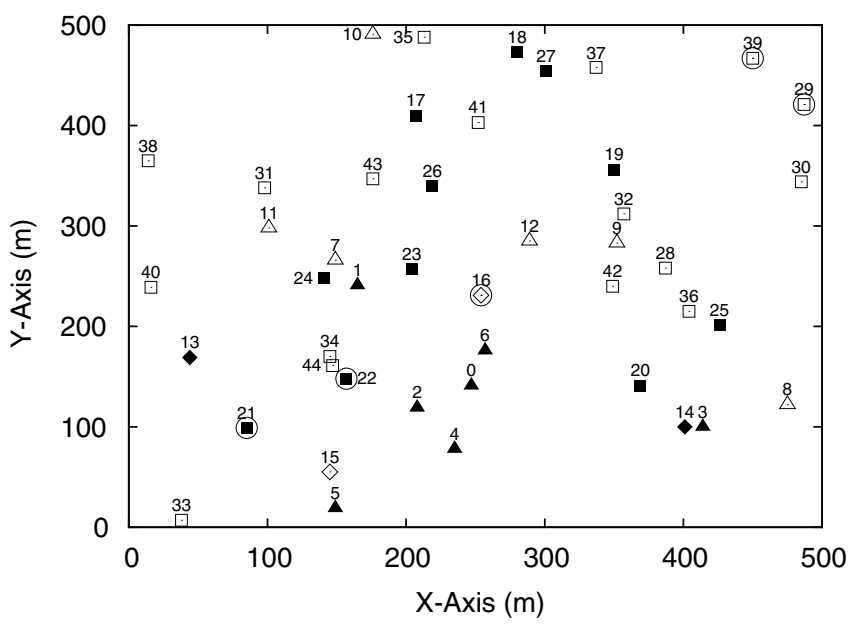

Fig. 10. Impact of network coordination (arbitrary bridge placement). (a) Throughput improvement; (b) placement of bridge nodes.

a

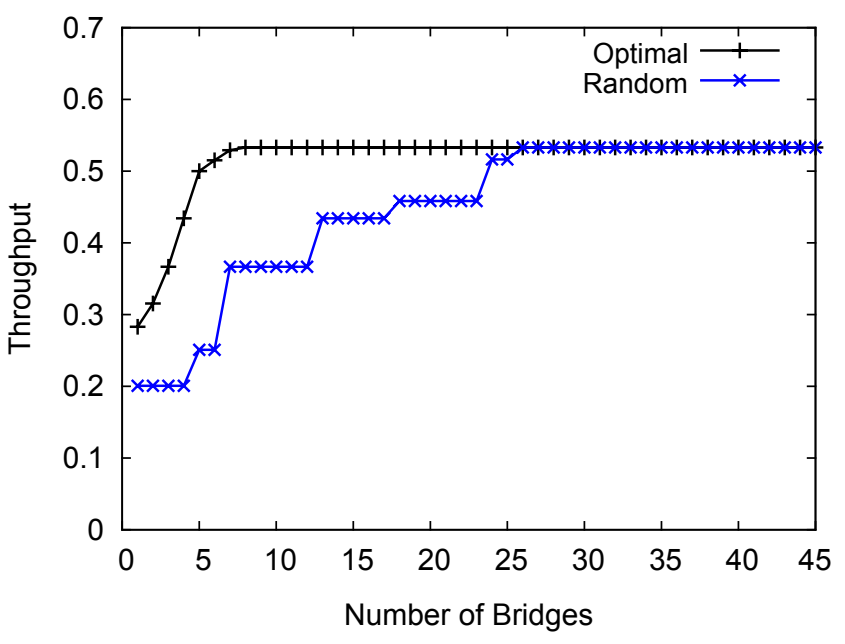

b

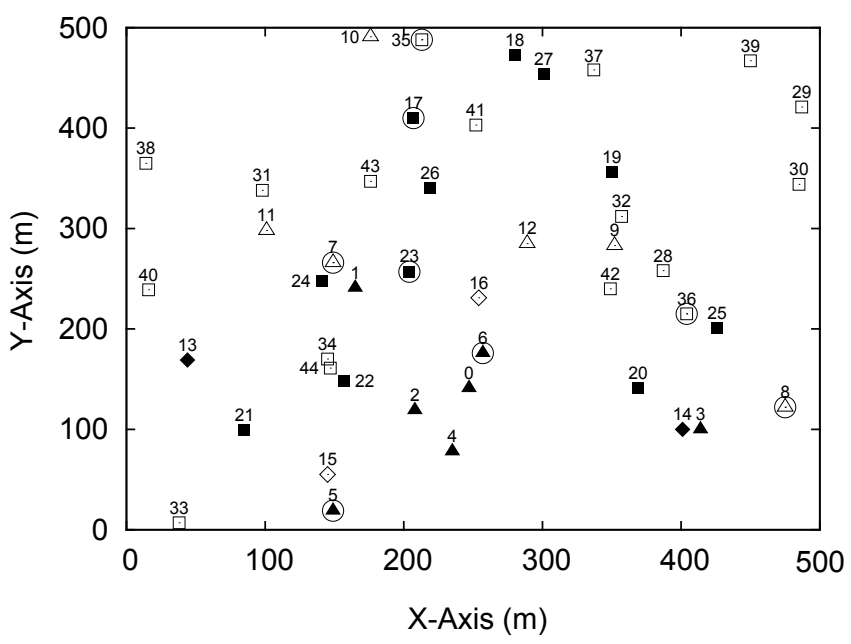

Fig. 11. Impact of network coordination (optimal bridge placement). (a) Throughput improvement; (b) placement of bridge nodes.

impact at all on reducing the wireless interference and improving the performance of the overall system. Consider, for example, the flat region in the lower bound curve when the number of bridges are between 7 and 12. Fig. 10(b) circles the five bridge nodes (from the 8th to the 12th bridge node selected) that do not contribute to overall throughput improvement. It can be observed from the figure that these bridge nodes are located either in a region too distant (in terms of the number of hops) from the source nodes to be helpful in diverting the traffic, or in a region already too crowded (too much contention) to relay traffic from another domain. It is clear that a cost-effective deployment of bridge nodes should take the node and traffic distributions of the overlaying networks into consideration.

To find the best bridge nodes for interworking, we include the constraint of bridge node placement (selection) in the LP formulation as described in Section 4. The performance gain due to arbitrary and optimal selection of bridge nodes is shown in Fig. 11(a) (only the lower bound is shown for clarity). It can be observed that if the placement of bridge nodes is chosen optimally, then only four bridge nodes are required for networks b1 and b2 to enjoy the performance gain due to network coordination (compared to throughputs in individual networks), and only eight bridge nodes are required for the overall system to achieve the optimal network capacity. Compared to 26 nodes in the random selection scenario, Fig. 11(a) substantiates the importance of bridge placement in interworking overlaying wireless mesh networks. The optimal bridge nodes that should be used to interwork networks b1 and b2 subject to the traffic distribution are nodes $6,7,23,5,8,35,17,36$ (in selection order). Fig. 11(b) shows the locations of these bridge nodes. It is interesting to note that the first bridge node (node 6 of network b1) selected is close to the gateway (node 16) of network 


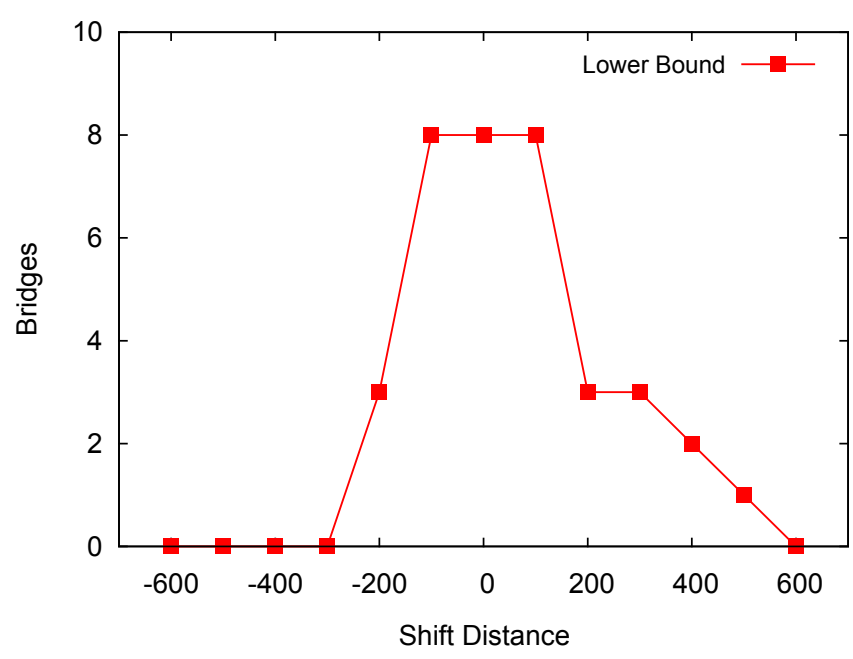

Fig. 12. Bridge nodes versus overlay region.

b2. It can be inferred that since the region around node 6 suffers from very high channel contention (four source nodes in the nearby), by making node 6 a bridge node, the traffic originated from the source nodes of network $b 1$ can be relayed to the nearby gateway provided by network b2 instead of relaying to the distant gateway nodes of network b1 at nodes 13 and 14. Nodes in network b1 thus benefit from interworking with network b2 and the overall network capacity is increased. That nodes 23 and 5 are chosen for inter-domain traffic relay is also due to the same reason.

In Fig. 12 we show the number of bridge nodes required to achieve the maximum network capacity versus the degree of overlap between overlaying mesh networks. Similar to that shown in Fig. 9, we shift the locations of nodes in network b1 to create scenarios with different degrees of overlapping between networks b1 and b2. It is clear that as the region of overlap decreases, the number of bridge nodes required decreases due to decreased interference between the two networks. An interesting point to note from Fig. 12 is that when the shift distance is between -600 and -300 , the number of bridge nodes required is zero. Alternatively, it does not help (in terms of maximum achievable throughput) to make existing mesh nodes as bridge nodes for relaying inter-domain traffic-even the source nodes of the two networks do suffer from throughput degradation as shown in Fig. 9(a). The reason can be inferred by reading Fig. 6 carefully. Network b1 has a gateway node (node 14) located at the right-hand side of the topology, while network b2 has a gateway node (node 15) located at the left-hand side of the topology. Therefore, when the shift distance is -300 , the two gateway nodes of the two networks are nearby, and hence it does not help to relay traffic from one network to another for accessing the gateway node of the other domain-especially since the region around the gateway nodes is already under high contention due to the increased interference. For shift distance less than -300 (e.g. from -600 to -300 ), it is not justified for nodes in, say, network b1 to use nodes in network b2 for accessing the gateway nodes of network b2 since such bridge nodes would have been more distant than the gateway node of network b1. Note that when the shift distance is between +400 and +600 , the required number of bridge nodes is not zero since the location of node 16 (gateway node of network b2) is further into the center of network 2 .

We use Fig. 13 to show that two heterogeneous, overlaying wireless mesh networks that do not necessarily interfere with each other can also benefit from the deployment of bridge nodes for interworking. We start with the network topology shown in Fig. 6 and replace network b2 with an 802.11a network a1 as shown in Fig. 13(a). We consider single-radio 802.11a/b bridge nodes that can switch between 802.11a and 802.11b modes for relaying inter-domain traffic. As described in Section 3, such bridge nodes are modeled as $N_{u}=\{(1,0,0),(0,1,0)\}$ and $K_{u}=2$. Since $802.11 \mathrm{a}$ and $802.11 \mathrm{~b}$ radios operate in different frequency bands, networks b1 and a1 do not interfere with each other. However, as Fig. 13(b) shows, the throughput of the overlaying networks still increases with the number of bridge nodes. The reason again can be attributed to the ability to access the gateway nodes of the other domain in shorter hops through bridge nodes.

We have so far presented the benefits (in terms of throughput improvement) that result from interworking of two overlay wireless mesh networks. In Fig. 14, we show a potential problem that needs to be addressed when interworking two networks-namely throughput unfairness. We start with the network topology shown in Fig. 6, and allow networks b1 and b2 to have different scaling factors (minimum traffic demands) $\gamma_{1}$ and $\gamma_{2}$ for maximizing the overall network capacity. Fig. 14(a) shows the values of $\gamma_{1}$ and $\gamma_{2}$ for three different scenarios: scenario b1,b2 for two stand-alone networks, scenario b1/b2 for overlaying without inter-domain relay, and scenario $\mathrm{b} 1+\mathrm{b} 2$ for overlaying with inter-domain relay. The values of $\gamma_{1}$ and $\gamma_{2}$ for scenarios b1,b2 and b2/b2 have been shown in Fig. 7(a) and Fig. 9(a) (for shift distance $=0$ ) already. For scenario b1 + b2, since inter-domain relay is allowed, it is possible that one network is completely starved (e.g. its source nodes are far from all gateway nodes) while the other network takes all network resource (i.e. exclusive access to gateway nodes) if maximizing the overall network capacity is the only goal. As shown in Fig. 14(a), while the system capacity for scenario b1 + b2 is highest among all three scenarios, network b2 is completely starved due to inter-domain relay. If such unfairness is not addressed, there might be no incentive at all for network $b 2$ to interwork with network $b 1$.

One feasible solution toward addressing the problem is to have traffic policing at the bridge nodes to limit the total outgoing or incoming traffic across the two domains. While the actual mechanism to implement such policy is beyond the scope of this paper, we show in Fig. 14(b) the impact of enforcing unfairness control on the overall system performance. We introduce a variable $K$, where $\gamma_{1}=K \gamma_{2}$, to control the throughput unfairness between networks b1 and b2. Fig. 14(b) shows that as the ratio $K$ becomes larger, the overall network throughput also gets higher. However, the curve is getting "saturated" toward large unfairness ratio. This implies that it is possible to decide on an acceptable degree of unfairness between two interworked networks without sacrificing significantly the 
a

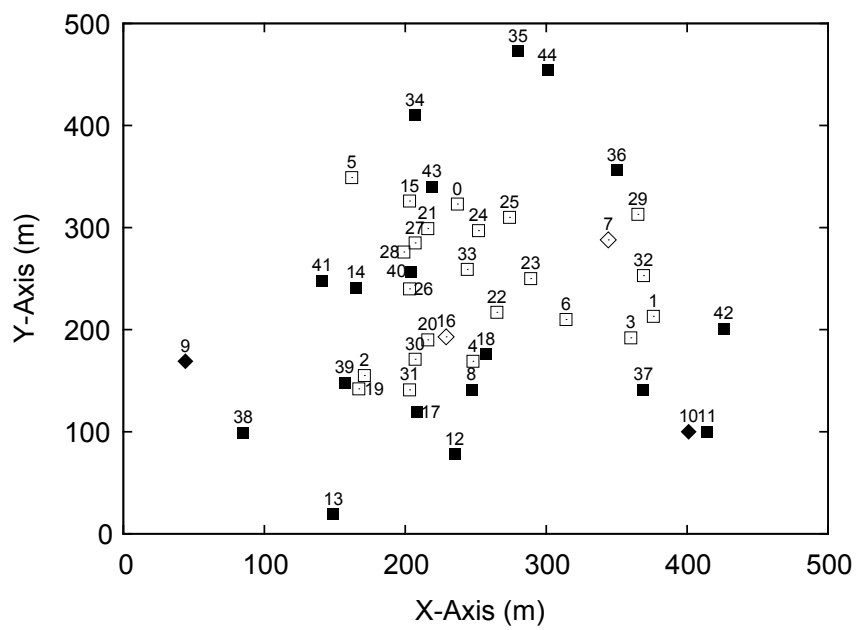

b

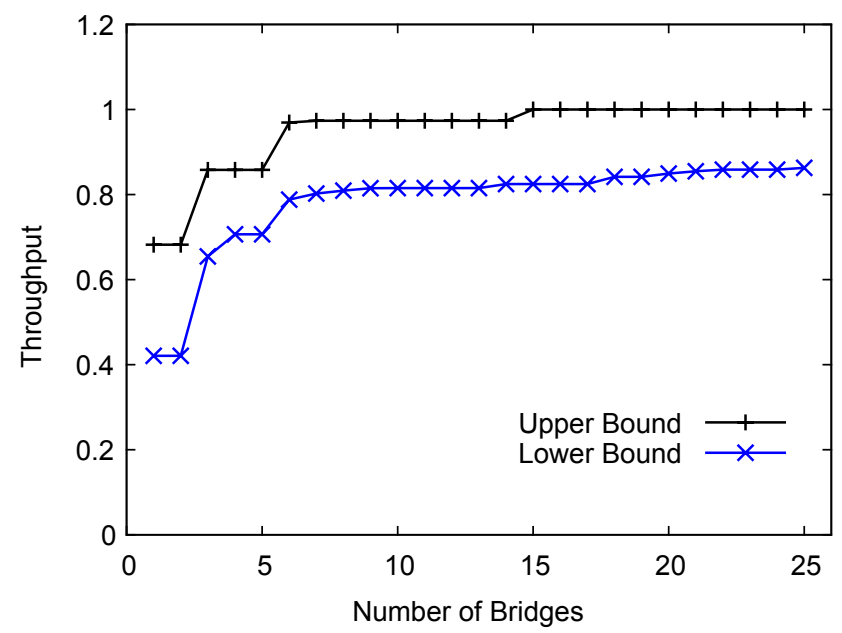

Fig. 13. Interworking 802.11a and 802.11b. (a) Node distribution; (b) throughput improvement.

a

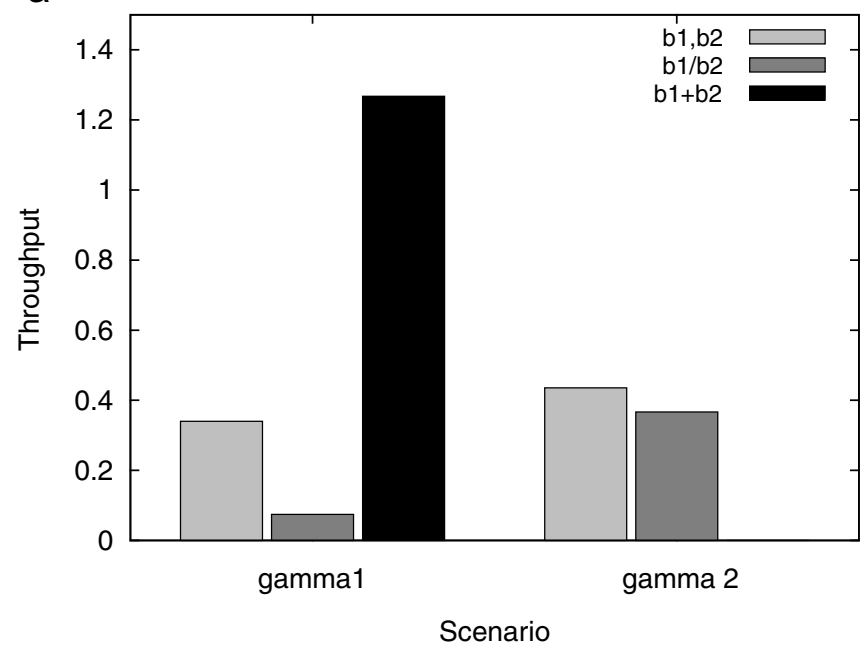

b

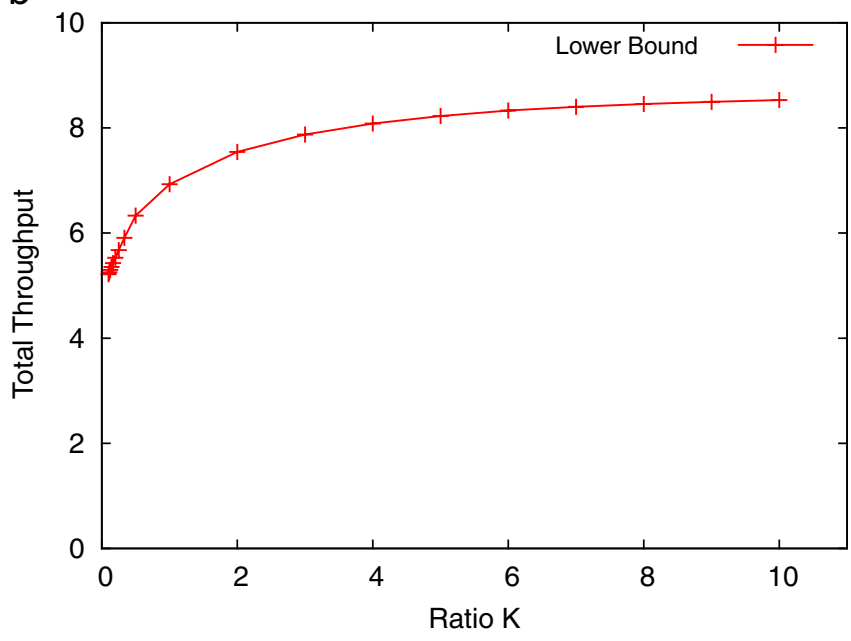

Fig. 14. Unfairness in interworking. (a) Throughput unfairness; (b) unfairness control.

performance of the overall system. Bandwidth allocation between overlaying wireless mesh networks thus needs to be carefully planned when enabling mesh network interworking.

\section{Conclusion}

In this paper, we investigate the impact when multiple wireless mesh networks overlap in service area. We formulate the problem of resource sharing as a network optimization problem, and present a general LP formulation for modeling the problem. We first find that in a system with multiple wireless mesh networks in overlap, if no form of coordination across domains is present, then individual mesh networks will suffer from significant capacity degradation. Therefore, it is desirable to "interwork" these wireless mesh networks by allowing interdomain traffic relay through provision of "bridge" nodes. If bridge nodes are provisioned without any planning, however, then serious unfairness problem may result. We then consider the problem of optimizing the selection of bridge nodes using minimal bridge nodes. Simulation results show that if proper interworking between overlaying mesh networks is provided, significant performance gain can be obtained.

\section{References}

[1] O. Aboul-Magd, et al., Joint SEE-Mesh/Wi-Mesh proposal to 802.11 TGs, IEEE 802.11-06/0328r0, February 2006.

[2] J. Agre, et al., Secure nomadic wireless mesh (SnowMesh) 802.11 TGs ESS mesh networking proposal, IEEE 802.11-05/596r1, May 2005.

[3] A. Akella, G. Judd, S. Seshan, P. Steenkiste, Self-management in chaotic wireless deployments, in: Proceedings of the 11th ACM Annual International Conference on Mobile Computing and Networking (MobiCom), Cologne, Germany, 2005, pp. 185-199. 
[4] M. Alicherry, R. Bhatia, L.E. Li, Joint channel assignment and routing for throughput optimization in multi-radio wireless mesh networks, in: Proceedings of the 11th ACM Annual International Conference on Mobile Computing and Networking (MobiCom), Cologne, Germany, 2005, pp. 58-72.

[5] Atheros WLAN Chipset Solutions, URL /http://www.atheros.com/pt/ index.html $\rangle$.

[6] J. Bicket, D. Aguayo, S. Biswas, R. Morris, Architecture and evaluation of an unplanned $802.11 \mathrm{~b}$ mesh network, in: Proceedings of the 11th ACM Annual International Conference on Mobile Computing and Networking (MobiCom), ACM Press, Cologne, Germany, 2005, pp. 31-42.

[7] V. Bychkovsky, B. Hull, A. Miu, H. Balakrishnan, S. Madden, A measurement study of vehicular internet access using in situ WiFi networks, in: Proceedings of the 12th ACM Annual International Conference on Mobile Computing and Networking (MobiCom), Los Angeles, CA, USA, 2006, pp. 50-61.

[8] A. Das, H. Alazemi, R. Vijayakumar, S. Roy, Optimization models for fixed channel assignment in wireless mesh networks with multiple radios, in: Proceedings of the Second IEEE Annual Communications Society Conference on Sensor and Ad Hoc Communications and Networks (SECON), Santa Clara, CA, USA, 2005, pp. 463-474.

[9] R. Draves, J. Padhye, B. Zill, Routing in multi-radio, multi-hop wireless mesh networks, in: Proceedings of the 10th ACM Annual International Conference on Mobile Computing and Networking (MobiCom), Philadelphia, PA, USA, 2004, pp. 114-128.

[10] P. Gupta, P. Kumar, The capacity of wireless networks, IEEE Trans. Inform. Theory 46 (2) (2000) 388-404.

[11] IEEE Computer Society, Part 11: Wireless LAN medium access control (MAC) and physical layer (PHY) specifications; Amendment 4: Further higher data rate extension in the $2.4 \mathrm{GHz}$ band, 2003.

[12] K. Jain, J. Padhye, V. Padmanabhan, L. Qiu, Impact of interference on multi-hop wireless network performance, in: Proceedings of the 9th ACM Annual International Conference on Mobile Computing and Networking (MobiCom), San Diego, CA, USA, 2003, pp. 66-80.

[13] M. Kodialam, T. Nandagopal, Characterizing the capacity region in multi-radio multi-channel wireless mesh networks, in: Proceedings of the 11th ACM Annual International Conference on Mobile Computing and Networking (MobiCom), Cologne, Germany, 2005, pp. 73-87.

[14] P. Kyasanur, N. Vaidya, Capacity of multi-channel wireless networks: impact of number of channels and interfaces, in: Proceedings of the 11th ACM Annual International Conference on Mobile Computing and Networking (MobiCom), Cologne, Germany, 2005, pp. 43-57.
[15] MeshDynamics Inc, URL 〈http://www.meshdynamics.com/〉.

[16] Microsoft mesh networking, URL 〈http://research.microsoft.com/mesh/ $\rangle$.

[17] MIT Roofnet, URL 〈http://pdos.csail.mit.edu/roofnet//.

[18] Nortel wireless mesh network solution, URL /http://www.nortel.com/ wirelessmesh>.

[19] J. Robinson, K. Papagiannaki, C. Diot, X. Guo, L. Krishnamurthy, Experimenting with a multi-radio mesh networking testbed, in: Proceedings of the First Workshop on Wireless Network Measurements (WiNMee), Trentino, Italy, 2005.

[20] Seattle Wireless, URL 〈http://www.seattlewireless.net/>.

[21] J. Tang, G. Xue, W. Zhang, Maximum throughput and fair bandwidth allocation in multi-channel wireless mesh networks, in: Proceedings of the 25th IEEE International Conference on Computer Communications (INFOCOM), Barcelona, Spain, 2006, pp. 1942-1951.

[22] N. Thompson, G. He, H. Luo, Flow scheduling for end-host multihoming, in: Proceedings of the 25th IEEE International Conference on Computer Communications (INFOCOM), Barcelona, Spain, 2006, pp. 1325-1336.

[23] Wireless Taipei-WIFLY, URL 〈http://www.wifly.com.tw/wifly3/en/ $\rangle$.

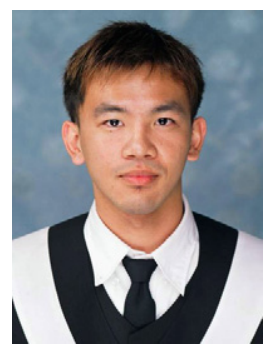

Tsai-Wei Wu received his B.S. degree in electrical engineering from Chang Gung University, Tao-Yuan, Taiwan, in ROC, 2004, and his M.S degree in communication engineering from $\mathrm{Na}$ tional Taiwan University, Taipei, Taiwan, ROC, in 2006. He is currently pursuing his $\mathrm{Ph} . \mathrm{D}$. degree in the Graduate Institute of Communication Engineering at National Taiwan University. His research interests include wireless mesh networks, wimax, and next generation wireless networks.

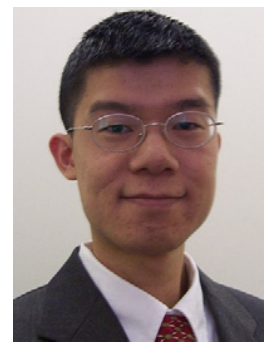

Hung-Yun Hsieh received the B.S. and M.S. degrees in electrical engineering from National Taiwan University, Taipei, Taiwan, ROC, and the Ph.D. degree in electrical and computer engineering from Georgia Institute of Technology, Atlanta, Georgia, USA. He joined the Department of Electrical Engineering and the Graduate Institute of Communication Engineering at National Taiwan University as an Assistant Professor in 2004. His research interests include wireless systems, mobile computing, and network protocols. 